\title{
Endoscopie et MICI : pourquoi mettre à jour les recommandations de la SFED?
}

\section{Endoscopy and IBD: why do we update the SFED recommendations?}

\author{
L. Peyrin-Biroulet \\ C Springer-Verlag France 2013
}

Poser le diagnostic de maladie inflammatoire chronique intestinale (MICI) peut parfois être difficile, même en 2013 à l'ère des biothérapies ! En effet, en dehors des granulomes qui sont pathognomoniques de la maladie de Crohn, le diagnostic de MICI repose le plus souvent sur un faisceau d'arguments cliniques, biologiques, radiologiques, endoscopiques et histologiques. L'apport de l'entéroscopie et de la vidéocapsule endoscopique nous a, entre autres, permis de reclasser certaines colites dites indéterminées en maladie de Crohn lorsqu'il existe, par exemple, une atteinte du grêle typique non visualisée par l'iléocoloscopie ni par les méthodes d'imagerie classiques telles que l'IRM.

Récemment, les objectifs thérapeutiques dans les MICI ont évolué, passant de l'obtention d'une rémission clinique au concept de cicatrisation muqueuse. Cicatriser la muqueuse de nos patients atteints de MICI est probablement le seul moyen de modifier l'histoire naturelle de leur maladie (réduction du nombre d'hospitalisations, du recours à la chirurgie, etc.). C'est ainsi que les scores endoscopiques sont de plus en plus souvent utilisés en pratique clinique. Toutefois, de nombreuses questions subsistent parmi lesquelles : faut-il systématiquement obtenir une cicatrisation muqueuse ? Quand faut-il réaliser l'endoscopie après avoir débuté le traitement immunosuppresseur par azathioprine et/ou anti-TNF (infliximab, adalimumab)?
Quel degré de cicatrisation muqueuse faut-il atteindre au cours de la maladie de Crohn et de la RCH ?

Près d'un patient sur dix présente une sténose intestinale lors du diagnostic de maladie de Crohn, et un nombre non négligeable de patients atteints de MICI vont présenter une sténose colique au cours du suivi. Après une résection iléocæcale, près d'un patient sur quatre avec une maladie de Crohn devra subir une seconde résection intestinale, le plus souvent pour une sténose anastomotique. La prise en charge de ces sténoses n'est pas toujours aisée, mais leur traitement endoscopique est de mieux en mieux codifié.

Enfin, laisser une inflammation muqueuse en place fait courir au patient un risque de dysplasie colorectale, et donc de cancer colorectal, même si ce risque a été revu récemment à la baisse (1,6\% dans la $\mathrm{RCH}$ après 14 ans de suivi). Avec la mise en place des programmes de surveillance endoscopique, il n'est pas rare de diagnostiquer un adénome chez un patient atteint de MICI (adénome sporadique, DALM [dysplasia associated lesion or mass], ALM [adenom-like mass]) et il n'est pas toujours facile de s'y retrouver dans cette nomenclature complexe qui présente pourtant un intérêt majeur en endoscopie, puisque la prise en charge de l'adénome en découlera directement.

Gageons que ces nouvelles recommandations aideront les endoscopistes que nous sommes à améliorer la prise de nos patients atteints de MICI. Bonne lecture!

L. Peyrin-Biroulet $(\square)$

Service d'hépatogastroentérologie, CHU de Nancy, allée du Morvan, F-54511 Vandœuvre-lès-Nancy, France e-mail : peyrinbiroulet@gmail.com 